

\title{
La structure cristalline de l'oxyfluorotantalate de potassium K2Ta2O3F6
}

Marcus Vlasse, Jean-Pierre Chaminade, Michel Pouchard

\section{To cite this version:}

Marcus Vlasse, Jean-Pierre Chaminade, Michel Pouchard. La structure cristalline de l'oxyfluorotantalate de potassium K2Ta2O3F6. Bulletin de la Société Française de Minéralogie et de Cristallographie, 1976, 99, pp.3-7. hal-00131278

\section{HAL Id: hal-00131278 \\ https://hal.science/hal-00131278}

Submitted on 10 Dec 2021

HAL is a multi-disciplinary open access archive for the deposit and dissemination of scientific research documents, whether they are published or not. The documents may come from teaching and research institutions in France or abroad, or from public or private research centers.
L'archive ouverte pluridisciplinaire HAL, est destinée au dépôt et à la diffusion de documents scientifiques de niveau recherche, publiés ou non, émanant des établissements d'enseignement et de recherche français ou étrangers, des laboratoires publics ou privés. 
Bull. Soc. fr. Minéral. Cristallogr.

(1976), 99, 3-7.

\title{
La structure cristalline de l'oxyfluorotantalate de potassium $\mathrm{K}_{2} \mathrm{Ta}_{2} \mathrm{O}_{3} \mathrm{~F}_{6}$
}

\author{
par Marcus Vlasse, Jean-Pierre CHAMinade et Michel POUCHARD, \\ Laboratoire de Chimie du Solide du C. N. R. S., Université de Bordeaux I ( $\left.{ }^{\mathbf{}}\right)$.
}

\begin{abstract}
Résumé. - La structure de l'oxyfluorotantalate de potassium $\mathrm{K}_{2} \mathrm{Ta}_{2} \mathrm{O}_{3} \mathrm{~F}_{6}$ a été déterminée à partir d'une synthèse de Patterson et affinée par la méthode des moindres carrés $(\mathrm{R}=\mathrm{0}, 065)$. La maille élémentaire de symétrie orthorhombique comporte le groupe spatial Pnma ( $a=$ I0,2 I2 (5) $\AA ; b=5,5^{81}$ (3) $\AA ; c=$ I 4,499 (7) $\AA$ ) et contient quatre motifs formulaires. Le réseau est formé de chaînes doubles se développant en zigzag dans la direction de l'axe $\mathbf{b}$, constituées d'octaèdres $\left(\mathrm{TaO}_{3} \mathrm{~F}_{3}\right)^{4-}$ mettant en commun trois sommets oxygénés d'une même face. Des ions $\mathrm{K}^{+}$occupent les sites vacants entre les files assurant la cohésion du réseau.
\end{abstract}

Mots clés : structure cristalline, oxyfluoro mixte, structure en chaîne, rayons X.

Crystal structure of potassium tantalum oxyfluoride $\mathrm{K}_{2} \mathrm{Ta}_{2} \mathrm{O}_{3} \mathrm{~F}_{6}$

Abstract. - The structure of potassium tantalum oxyfluoride $\mathrm{K}_{2} \mathrm{Ta}_{2} \mathrm{O}_{3} \mathrm{~F}_{6}$ has been determined from a Patterson synthesis and refined by least squares $(\mathrm{R}=0.065)$. The orthorhombic unit cell has the space group Pnma ( $a=\operatorname{I0.2I2}(5) \AA ; b=5.58$ I (3) $\AA$; $c=14.499$ (7) $\AA$ ) and contains four motifs. The structure is made up of double infinite zigzag chains along the [oro] direction. These chains are formed by the $\left(\mathrm{TaO}_{3} \mathrm{~F}_{3}\right)^{4-}$ octahedra sharing three oxygen corners of a common face. The $\mathrm{K}^{+}$ions occupy the vacant sites between the files and ensure the cohesion of the lattice.

Key words : crystal structure, mixed oxyfluoride, chain structure, X-rays.

\section{INTRODUCTION.}

Dans le cadre de l'étude entreprise au laboratoire des systèmes élément alcalin (ou argent) tantale $(+\mathrm{V})$ - oxygène - fluor (Pocuhard et al. I972 ; Chaminade et al., I972 $a, b$; Chaminade, I974; Vlasse et al., I973, I975 $a, b$ ) et plus particulièrement du système $\mathrm{K}-\mathrm{Ta}-\mathrm{O}-\mathrm{F}$, quatre oxyfluorotantalates avaient été isolés: $\mathrm{K}_{2} \mathrm{Ta}_{4} \mathrm{O}_{9} \mathrm{~F}_{4}, \mathrm{~K}_{3} \mathrm{TaO}_{2} \mathrm{~F}_{6}$, et $\mathrm{K}_{2} \mathrm{Ta}_{2} \mathrm{O}_{3} \mathrm{~F}_{6}$ (Chaminade et al., I974). Les structures des trois premières phases ont été précisées, la dernière, $\mathrm{K}_{2} \mathrm{Ta}_{2} \mathrm{O}_{3} \mathrm{~F}_{6}$, est l'objet de ce mémoire.

Lors de l'étude du système K-Ta-O-F, nous avions vainement tenté de repréparer le sel de Marignac formulé $\mathrm{K}_{4} \mathrm{Ta}_{4} \mathrm{O}_{5} \mathrm{~F}_{14}$ par plusieurs auteurs (Marignac, I866; Ts'ui Ping-Hsin et al., I963; Buslaev et al., I965; Davidovitch et al., I972). Ces auteurs pensaient obtenir $\mathrm{K}_{4} \mathrm{Ta}_{4} \mathrm{O}_{5} \mathrm{~F}_{14}$ par hydrolyse de l'heptafluorotantalate de potassium $\mathrm{K}_{2} \mathrm{TaF}_{7}$.

L'étude radiocristallographique d'échantillons

(I) 35I, cours de la Libération, 33405 Talence. préparés par voie sèche et correspondant à la composition $\mathrm{K}_{4} \mathrm{Ta}_{4} \mathrm{O}_{5} \mathrm{~F}_{14}$ révèla en fait la présence de deux phases, le fluorotantalate $\mathrm{KTaF}_{6}$ et une phase que nous avons isolée avec la composition $\mathrm{K}_{2} \mathrm{Ta}_{2} \mathrm{O}_{3} \mathrm{~F}_{6}$. Le spectre de diffraction $\mathrm{X}$ de cette dernière correspond en faî à celui précédemment attribué au sel de Marignac, dont on peut ainsi mettre en doute la composition avancée.

Il nous a donc paru intéressant de poursuivre notre étude des composés oxyfluorés du tantale et de résoudre l'ambiguité existant entre le sel de Marignac et $\mathrm{K}_{2} \mathrm{Ta}_{2} \mathrm{O}_{3} \mathrm{~F}_{6}$ par une détermination structurale complète de cette phase.

\section{Préparation.}

$\mathrm{K}_{2} \mathrm{Ta}_{2} \mathrm{O}_{3} \mathrm{~F}_{6}$ a été préparé par interaction de quantités calculées de pentoxyde de tantale $\mathrm{Ta}_{2} \mathrm{O}_{5}$, de tantalate de potassium $\mathrm{KTaO}_{3}$ et d'heptafluorotantalate de potassium $\mathrm{K}_{2} \mathrm{TaF}_{7}$. La synthèse a été effectuée en tube d'or scellé sous argon à une température de $700^{\circ} \mathrm{C}$. Un traitement thermique à $750^{\circ} \mathrm{C}$, suivi d'un refroidissement lent, a permis l'obtention de monocristaux de $\mathrm{K}_{2} \mathrm{Ta}_{2} \mathrm{O}_{3} \mathrm{~F}_{9}$. 


\section{ÉTUDE RADIOCRISTALLOGRAPHIOUE.}

L'examen des diagrammes de diffraction $\mathrm{X}$, obtenus à l'aide des méthodes de Weissenberg et de Buerger, a permis de déterminer une symétrie orthorhombique pour la phase $\mathrm{K}_{2} \mathrm{Ta}_{2} \mathrm{O}_{3} \mathrm{~F}_{6}$. Les paramètres de la maille sont les suivants :

$$
\begin{aligned}
& a=\mathrm{I}, 2 \mathrm{I} 5 \pm 0,005 \AA \\
& b=5,58 \mathrm{I} \pm 0,003 \AA \\
& c=\mathrm{I} 4,499 \pm 0,007 \AA
\end{aligned}
$$

Deux conditions d'existence ont été relevées :

$$
\begin{aligned}
& o k l: k+l=2 n \\
& h k \circ: h=2 n,
\end{aligned}
$$

ce qui laisse le choix entre deux groupes d'espace : Pnma et Pna2 .

La masse volumique mesurée $\left(\rho_{e x p}=4,794 \pm\right.$ $0,005 \mathrm{~g} \cdot \mathrm{cm}^{-3}$ ) est en bon accord avec la masse volumique calculée $\left(p_{c a l c}=4,835 \mathrm{~g} \cdot \mathrm{cm}^{-3}\right)$ pour un nombre de motifs par maille $Z=4$.

Un monocristal en forme de prisme liexagonal de très petites dimensions $\left(0,03 \times 0,03 \times 0,04 \mathrm{~mm}^{3}\right)$ a été utilisé pour l'enregistrement des intensités de diffraction $\mathrm{X}$. Ces intensités ont été mesurées à l'aide d'un diffractomètre automatique EnrafNonius CAD-3 à trois cercles, elles sont obtenues avec la radiation monochromatique $\mathrm{MoK} \alpha$ $\left(\lambda=0,7\right.$ IO7 $\AA$ ) jusqu'à $\theta_{\max }=40^{\circ}$ (balayage $\theta-2 \theta)$.

La très petite taille du cristal permet de limiter les effets d'absorption $\left(\mu r_{\max }=0,6\right)$. L'examen des réflexions équivalentes d'un certain nombre de taches indique une variation en intensité de $2 \%$ à $5 \%$.
Le cristal étant orienté selon l'axe b, les intensités ont été mesurées pour un quart de l'espace réciproque donnant I 626 réflexions indépendantes. Cet enregistrement a été corrigé de l'effet Lorentzpolarisation.

Les facteurs de diffusion atomique de l'oxygène, du fluor, du potassium et du tantale sont ceux donnés par Mc Master et al. (I969).

\section{RÉsolution et AfFinement de LA STRUcture.}

Les positions des atomes de tantale et de potassium ont été déterminées à partir d'un calcul tridimensionnel de la fonction de Patterson. Sur la base d'un calcul de distribution statistique des intensités (test de Srinivasan, I96o) qui indique une distribution centrosymétrique, nous avons retenu le groupe Pnma. En outre, les intensités de réflexions $h k l$ (fortes) avec $k$ de même parité montrent une décroissance semblable en fonction de $\sin \theta / \lambda$. Ceci corrobore le groupe centrosymétrique. Dans ce groupe, les atomes lourds occupent la position :

$$
4(c):(x, I / 4, z)
$$

Quelques cycles d'affinement permettent d'abaisser le facteur $R$ jusqu'à la valeur o,I4. Une carte de densité électronique, déterminée par transformée de Fourier, permet de localiser les anions en :

$$
4(c):(x, I / 4, z) \text { et } 8(d):(x, y, z) .
$$

Plusieurs cycles d'affinement ont alors été effec-

\begin{tabular}{|c|c|c|c|c|c|c|c|c|c|}
\hline Atonie & $\mathrm{x}$ & $y$ & $z$ & ${ }^{8} 11$ & ${ }^{\beta} 22$ & $\beta_{33}$ & $\beta_{12}$ & $\beta_{13}$ & $B_{23}$ \\
\hline $\mathrm{Ta}(1)$ & $0,0660(1)$ & $1 / 4$ & $0,1492(1)$ & $0,00154(7)$ & $0,00658(26)$ & $0,00090(4)$ & 0 & $0,00003(4)$ & 0 \\
\hline $\mathrm{Ta}(2)$ & $0,7814(1)$ & $1 / 4$ & $0,9915(1)$ & $0,00138(7)$ & $0,00618(26)$ & $0,00096(3)$ & 0 & $0,00015(5)$ & 0 \\
\hline$K(1)$ & $0,4405(7)$ & $1 / 4$ & $0,0983(5)$ & $0,00280(48)$ & $0,01694(20 s)$ & $0,00151(20)$ & 0 & $0,00072(30)$ & 0 \\
\hline$K(2)$ & $0,7392(9)$ & $1 / 4$ & $0,7082(6)$ & $0,00650(83)$ & $0,01930(265)$ & $0,00270(37)$ & 0 & $0,00004(5)$ & 0 \\
\hline$F(1)$ & $0,2308(21)$ & $1 / 4$ & $0,2191(14)$ & $0,00315(153)$ & $0,04606(950)$ & $0,00142(77)$ & 0 & $-0,00243(107)$ & 0 \\
\hline$F(2)$ & $0,1413(13)$ & $1 / 4$ & $0,6030(17)$ & $0,00682(254)$ & $0,03434(923)$ & $0,00282(112)$ & 0 & $0,00188(79)$ & 0 \\
\hline$F(3)$ & $0,0102(9)$ & $0,4869(15)$ & $0,2403(10)$ & $0,00651(172)$ & $0,01855(467)$ & $0,00302(70)$ & $0,00134(124)$ & $0,00159(89)$ & $-0,001.88(83)$ \\
\hline$F(4)$ & $0,1681(13) \mid$ & $0,4800(14)$ & $0,4466(9)$ & $0,00430(121)$ & $0,01897(453)$ & $0,00267(61)$ & $0,00426(203)$ & $-0,00041(36)$ & $0,00227(143)$ \\
\hline$O(1)$ & $0,3950(10)$ & $1 / 4$ & $0,4066(15)$ & $0,00302(164)$ & $0,04379(838)$ & $0,00252(98)$ & 0 & $0,00036(40)$ & 0 \\
\hline$O(2)$ & $0,1372(18)$ & $0,5003(18)$ & $0,0741(12)$ & $0,00814(178)$ & $0,02309(608)$ & $0,00420(87)$ & $-0,00067$ (92) & $0,00177(103)$ & $0,00085(52)$ \\
\hline
\end{tabular}
tués sur la base de diverses distributions anioniques oxygène-fluor. Ceci a été réalisé en permutant la

TABLEAU $I$.

\section{Coordonnées réduites et facteurs d'agitation thermique anisotrope pour $K_{2} T_{2} \mathrm{O}_{3} \mathrm{~F}_{6}$.}

(Les déviations standards portant sur la dernic̀re décimale sont données par les chiffres entre parenthèses; le facteur d'agitation thermique est égal à : $\exp \left[-\mathrm{IO}^{-5}\left(h^{2} \beta_{11}+k^{2} \beta_{22}+l^{2} \beta_{33}+2 h l \beta_{13}+2 k l \beta_{23}\right]\right.$.) 
position de l'anion $\mathrm{O}(\mathrm{I})$ avec celle de $\mathrm{F}(\mathrm{I})$ ou $\mathrm{F}(2)$, et la position de l'anion $\mathrm{O}(2)$ avec celle de $\mathrm{O}(3)$ ou $\mathrm{O}(4)$ ainsi qu'avec une distribution statistique des anions, tout autre paramètre restant identique. L'ordre oxygène-fluor correspondant au tableau I a pu ainsi être retenu grâce à un facteur $\mathrm{R}$ notablement plus faible pour cette distribution. L'introduction des facteurs d'agitation thermique anisotrope des divers atomes a permis, en outre, d'abaisser le facteur $R$ jusqu'à la valeur 0,065 , pour le groupe Pnma avec I 396 réflexions.

L'affinement a été effectuée grâce à un algorithme des moindres carrés utilisant une matrice complète (Busing et al., I962), modifié pour permettre une correction de dispersion anormale.

Nous avons minimisé ainsi l'expression $\Sigma \omega$ $\left\|\mathrm{F}_{o}-\mathrm{F}_{c}\right\|^{2}$ où $\mathrm{F}_{o}$ et $\mathrm{F}_{c}$ sont les amplitudes des facteurs de structure observés et calculés et $\omega$ la prépondérante égale à I pour toutes réflexions. Un facteur d'échelle générale a été utilisé durant tout l'affinement.

Les coordonnées réduites des atomes et les facteurs d'agitation thermique anisotrope sont rassemblés au tableau I (1)

DEscription ET DISCUSSION DE LA STRUCTURE.

La projection de la structure sur le plan (oIo) est représentée à la figure $\mathrm{I}$.



FIG. I. - Projection de la structure de $\mathrm{K}_{2} \mathrm{Ta}_{2} \mathrm{O}_{3} \mathrm{~F}_{6}$ sur le plan (oro).

L'environnement anionique des atomes de tantale consiste en octaèdres légèrement déformés, comme l'indiquent les distances et angles interatomiques rassemblés au tableau II.

Ces octaèdres $\left(\mathrm{TaO}_{3} \mathrm{~F}_{3}\right)^{4-}$, liés par des sommets communs en position cis, forment une chaîne en zigzag de composition $\left(\mathrm{TaO}_{2} \mathrm{~F}_{3}\right)_{n}{ }^{2 n-}$ qui se développe parallèlement à l'axe b (fig. $2 b$ ).

(I) La liste des facteurs de structure observés et calculés peut être communiquée par les auteurs.
TABI.EAU II.

Distances et angles interatomiques

dans $\mathrm{K}_{2} \mathrm{Ta}_{2} \mathrm{O}_{3} \mathrm{~F}_{6}$ en $\AA( \pm 0,006 \AA)$ et en degrés $\left( \pm 0,05^{\circ}\right)$.
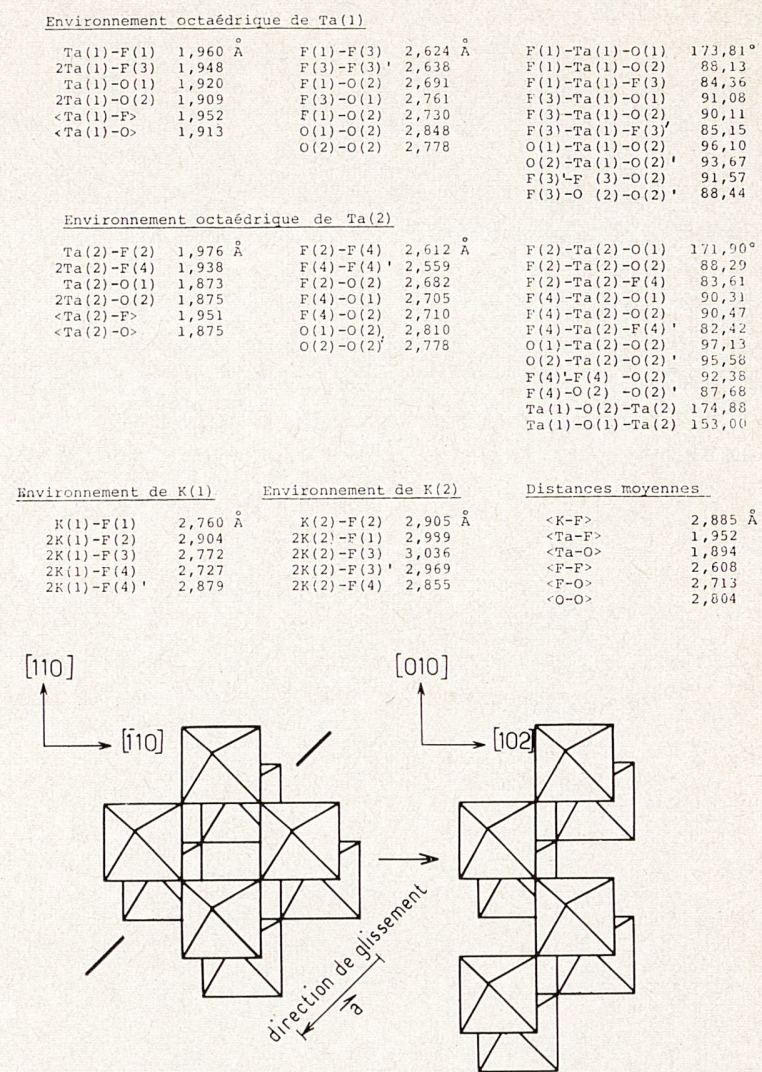

$\mathrm{Re} \mathrm{O}_{3}$

File infinie d'octaedres le long del'axe $\vec{b}$ de $\mathrm{K}_{2} \mathrm{Ta}_{2} \mathrm{O}_{3} \mathrm{~F}_{6}$

(a)

(b)

FIG. 2. - Relation entre la maille de $\mathrm{ReO}_{3}$ et une file d'octaèdres dans $\mathrm{K}_{2} \mathrm{Ta}_{2} \mathrm{O}_{3} \mathrm{~F}_{6}$.

Une telle chaîne est accolée dans le plan (ĪOI) à une seconde chaîne identique grâce à des sommets communs; deux chaînes forment ainsi une file infinie selon la direction [OIO], que l'on peut formuler $\left(\mathrm{Ta}_{2} \mathrm{O}_{3} \mathrm{~F}_{6}\right)_{n}{ }^{2 n-}$. Les atomes de potassium sont insérés entre ces files, assurant la cohésion du réseau (fig. I).

Ces files peuvent être également décrites à partir d'une maille unitaire $\mathrm{ReO}_{3}$ (fig. 2), en faisant glisser deux octaèdres $\mathrm{ReO}_{3}$ dans la direction [IOO] (vecteur a), de manière à donner naissance à un assemblage d'octaèdres identique à celui trouvé dans $\mathrm{K}_{2} \mathrm{Ta}_{2} \mathrm{O}_{3} \mathrm{~F}_{6}$.

Une distribution ordonnée des atomes d'oxygène et de fluor a été mise en évidence. Les atomes d'oxygène occupent les sommets communs des octaèdres $\left(\mathrm{TaO}_{3} \mathrm{~F}_{3}\right)^{4-}$; autrement dit, les atomes d'oxygène sont toujours liés à deux atomes de tantale, formant les chaînes infinies Ta-O-Ta-O-Ta, 
parallèles à l'axe b. Les atomes de fluor, par contre, occupent les sommets non liés des octaèdres et constituent, en outre, les polyèdres de coordination du potassium (fig. 3).

La distance moyenne $<\mathrm{Ta}-\mathrm{O}\rangle=\mathrm{I}, 894 \AA$ est nettement plus courte que la somme des rayons ioniques $<\mathrm{Ta}-\mathrm{O}\rangle_{\text {theor. }}=2,04 \AA$ (Shannon et Prewitt, I969), elle est plus courte que la distance moyenne $<$ Ta-F $>=\mathrm{I}, 952 \AA\left(<\mathrm{Ta}-\mathrm{F}>_{\text {théor. }}=\right.$ I,97 $\AA$ ) (tableau II).

En revanche, les liaisons F-F, F-O, O-O, de distance moyenne $2,608 \AA, 2,7 \mathrm{I} 3 \AA$ et $2,804 \AA$ respectivement, sont en bon accord avec la somme des rayons ioniques $(\mathrm{F}-\mathrm{F}=2,66 \AA, \mathrm{F}-\mathrm{O}=2,73 \AA$, $\mathrm{O}-\mathrm{O}=2,80 \AA$ ) et avec les liaisons F-F, F-O, O-O généralement observées dans les oxyfluoroniobates ou oxyfluorotantalates alcalins (Lundberg, I965; Anderson et Galy, I969; Vlasse et al., I973, I975 $a, b$ ). De même, les liaisons K-F au sein du polyèdre de

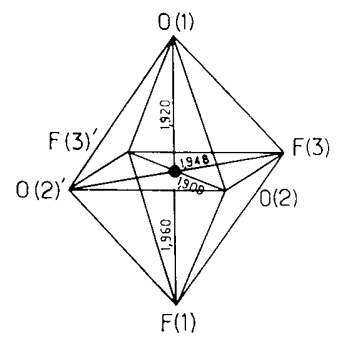

$\mathrm{Ta}(1)$

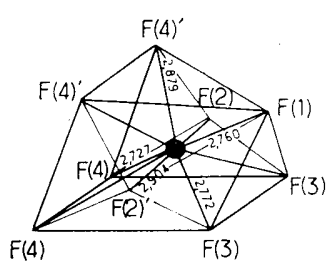

$K(1)$

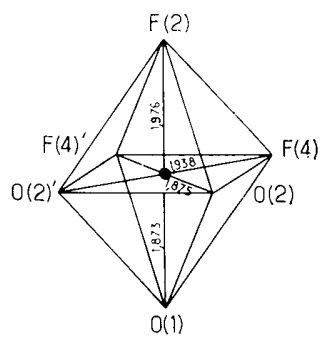

$\mathrm{Ta}(2)$

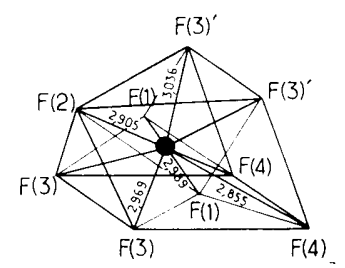

$K(2)$
FIG. 3. - Polyèdres de coordination du potassium et du tantale (les distances interatomiques sont en $\AA$ ).

coordination fluoré du potassium (coordinence 9), de distance moyenne $2,885 \AA$, sont compatibles avec la somme des rayons ioniques $(2,88 \AA)$ et avec les liaisons $\mathrm{K}-\mathrm{F}$ observées dans l'heptafluoroniobate de potassium $\mathrm{K}_{2} \mathrm{NbF}_{7}$ (distances de 2,65 à 2,94 $\AA$ )
(Hoard, I939) où le potassium se trouve également en coordinence 9 .

L'écart observé entre les longueurs des liaisons Ta-O dans $\mathrm{K}_{2} \mathrm{Ta}_{2} \mathrm{O}_{3} \mathrm{~F}_{6}$ et la somme des rayons ioniques montre que les octaèdres sont fortement liés les uns aux autres par des liaisons Ta-O à caractère covalent très marqué. Une étude des spectres d'absorption infrarouge menée par Kharitonov et Buslaev (I964) sur le "sel de Marignac " a mis en évidence la présence de chaînes -Ta-O-Ta-O- ainsi qu'un caractère de double liaison entre atomes de tantale et d'oxygène. Ce résultat conforte notre propre étude et permet de justifier l'apparente contradiction entre les distances $\mathrm{Ta}-\mathrm{O}$ et $\mathrm{Ta}-\mathrm{F}$ observées. En effet, dans les chaînes doubles de l'oxyfluorure $\mathrm{K}_{2} \mathrm{Ta}_{2} \mathrm{O}_{3} \mathrm{~F}_{6}$, chaque atome de tantale disposant de trois orbitales atomiques $5 d\left(t_{2 g}\right)$ est situé au voisinage de trois atomes d'oxygène, eux-mêmes entourés chacun de deux atomes de tantale. Chaque oxygène dispose donc, comme dans la maille de $\mathrm{ReO}_{3}$, de deux orbitales $2 p$ susceptibles de donner une double liaison de type $\pi$ avec chacun de ses deux voisins. Il s'en suit la formation d'un véritable réseau cationique monodimensionnel $\left(\mathrm{Ta}_{2} \mathrm{O}_{3}\right)^{4+}$, que l'on peut comparer aux chaînes $(\mathrm{TiO})_{n}{ }^{2 n+}$ présents dans des sels de titanyles tels que $\mathrm{TiOSO}_{4} \cdot \mathrm{H}_{2} \mathrm{O}$ (Lundgren, r956) et qui met en évidence le caractère acide du Ta (V).

\section{CONCLUSIONS.}

La structure cristalline de $\mathrm{K}_{2} \mathrm{Ta}_{2} \mathrm{O}_{3} \mathrm{~F}_{9}$ a été déterminée, permettant ainsi de lever l'ambiguité concernant la formulation du sel de Marignac. Une distribution ordonnée des atomes d'oxygène et de fluor a été mise en évidence. Les atomes d'oxygène assurent les liaisons entre octaèdres voisins pour former des chaînes doubles infinies O-Ta-O-Ta-O de formulation $\left(\mathrm{Ta}_{2} \mathrm{O}_{3}\right)^{4+}$. Les atomes de fluor occupent les autres sommets des octaèdres de coordination du tantale et constituent ceux du potassium. La mise en évidence de distances Ta-O ayant un caractère de double liaison entre tantale et oxygène est en parfait accord avec les résultats obtenus par absorption IR par Kharitonov et Buslev (I964). Elle confirme l'originalité de cette structure.

Reçu le 3 décembre 1975. Accepté le 25 février 1976.

\section{BIBLIOGRAPHIE}

Anderson, S. et Galy, J. (1969). - Acta Cryst., $B 26,847$.

Busing, W. R., Martin, K. O. et Lívy, H. A. (I962). - ORFLS, ORNL, TM, 305, Oak Ridge National.

Buslaev, Yu. A., Davidovitch, R. L. et BochikaREVA, V. A. (r965). - Bull. Acad. Sc., U. R. S. S. Inorg. Materials, $1,445$.
Chaminade, J. P. (1974). - - Thèse de doctorat ès sciences, Bordeaux.

Chaminade, J. P., Pouchard, M. et HagenmulLER, P. (1972 a). - Rev. Chim. Minér., 9, 38I.

Chaminade, J, P., Vlasse, M., Pouchard, M. et Hagenmuller, P. (1972 b). - C. R. Acad. Sc., 277, II4I. 
Chaminade, J. P., Vilasse, M., Pouchard, M. et Hagenmuller, P. (1974). - Bull. Soc. Chem., 9-IO, I79I.

Davidovitch, T. A., Kaidalova, T. A., Levtchichina, T. F. et Sergienko, V. J. (I972). - Atlas des spectres d'absorption IR et données radiocristallographiques des complexes des fluorures des métaux des groupes IV et V, Édition "Naouka ", Moscou.

HoARd, J. L. (I939). - J. Amer. Chem. Soc., 6. I, I252. Kharitonov, V. G., Buslaev, Yu. A. (I964). - Izv. AN S. S. S. R., Ser. Khim., 808.

Lundberg, M. (I965). - Acta Chem. Scand., I9, 2274.

Lundgrenn, G. (I956). - Avkiv. kemi, I0, 397.

Mc Master, W. H., Kerr del Grande, N., Mallet, J. H. et Hubber, J. H. (1969). - Natl. Bur. Stand. Compilation of X-ray Cross Sections UCRL - 50I74, Sec. II, Rev. I.
Marignac, M. C. (r866). -- Ann. Chim. Phys., 9, 249. Pouchard, M. et Chamirade, J. P. (1972). - C. R. Acad. Sc., 274, I739.

Shannon, R. D. et Prewitt, C. T. (i969). - Acta Cryst., $B$ 25, 925.

Srinivasan, R. (1960). - Acta Cryst., I3, 388.

Ts'ui Ping-Hsin, Konstantinov, V. I. et LuzhNAYA, N. P. (1963). - Russ. J. Inorg. Chem., 8, 204.

Vlasse, M., Chaminade, J. P. et Pouchard, M. (1973). - Mat. Res. Bull., 8, II 7.

Vilasse, M., Chaminade, J. P., Massies, J. C. et Pouchard, M. (I975 a). - J. Solid State Chem., I2, IO2.

Vlasse, M., Moriliere, C., Chaminade, J. P. et Pouchard, M. (1975 b). - Bull. Soc. fr. Minéral. Cristallogr., 98, 325 . 
